\title{
Use of Quantitative Morphological and Functional Features for Assessment of Axillary Lymph Node in Breast Dynamic Contrast-Enhanced Magnetic Resonance Imaging
}

\author{
Roberta Fusco $\mathbb{D}^{1},{ }^{1}$ Mario Sansone $\mathbb{D}^{\mathbb{D}},{ }^{2}$ Vincenza Granata ${ }^{(D},{ }^{1}$ Maurizio Di Bonito, ${ }^{3}$ \\ Franca Avino, ${ }^{4}$ Orlando Catalano, ${ }^{1}$ Gerardo Botti, ${ }^{3}$ and Antonella Petrillo $\mathbb{D}^{1}$ \\ ${ }^{1}$ Radiology Unit, "Dipartimento di Supporto ai Percorsi Oncologici Area Diagnostica, Istituto Nazionale Tumori-IRCCS-Fondazione \\ G. Pascale", Via Mariano Semmola, Naples, Italy \\ ${ }^{2}$ Department of Electrical Engineering and Information Technologies, University "Federico II" of Naples, Via Claudio, Naples, Italy \\ ${ }^{3}$ Pathology Unit, "Dipartimento di Supporto ai Percorsi Oncologici Area Diagnostica, Istituto Nazionale Tumori-IRCCS-Fondazione \\ G. Pascale”, Via Mariano Semmola, Naples, Italy \\ ${ }^{4}$ Senology Surgery Unit, "Dipartimento Corp-S Assistenziale e di Ricerca dei Percorsi Oncologici del Distretto Toracico, Istituto \\ Nazionale Tumori-IRCCS-Fondazione G. Pascale”, Via Mariano Semmola, Naples, Italy
}

Correspondence should be addressed to Roberta Fusco; r.fusco@istitutotumori.na.it

Received 10 December 2017; Accepted 29 April 2018; Published 30 May 2018

Academic Editor: Giuseppe Falco

Copyright (C) 2018 Roberta Fusco et al. This is an open access article distributed under the Creative Commons Attribution License, which permits unrestricted use, distribution, and reproduction in any medium, provided the original work is properly cited.

\begin{abstract}
Background. Axillary lymph-node assessment is considered one of the most important prognostic factors concerning breast cancer survival. Objective. We investigated the discriminative power of morphological and functional features in assessing the axillary lymph node. Methods. We retrospectively analysed data from 52 consecutive patients who undergone DCE-MRI and were diagnosed with primary breast carcinoma: 94 lymph nodes were identified. Per each lymph node, we extracted morphological features: circularity, compactness, convexity, curvature, elongation, diameter, eccentricity, irregularity, radial length, entropy, rectangularity, roughness, smoothness, sphericity, spiculation, surface, and volume. Moreover, we extracted functional features: time to peak (TTP), maximum signal difference (MSD), wash-in intercept (WII), wash-out intercept (WOI), wash-in slope (WIS), washout slope (WOS), area under gadolinium curve (AUGC), area under wash-in (AUWI), and area under wash-out (AUWO). Selection of important features in predicting metastasis has been done by means of receiver operating characteristic (ROC) analysis. Performance of linear discriminant analysis was analysed. Results. All morphological features but circularity showed a significant difference between median values of metastatic lymph nodes group and nonmetastatic lymph nodes group. All dynamic parameters except for MSD and WOS showed a statistically significant difference between median values of metastatic lymph nodes group and nonmetastatic lymph nodes group. Best results for discrimination of metastatic and nonmetastatic lymph nodes were obtained by AUGC (accuracy 75.8\%), WIS (accuracy 71.0\%), WOS (accuracy 71.0\%), and AUCWO (accuracy 72.6\%) for dynamic features and by compactness (accuracy $82.3 \%$ ), curvature (accuracy $71.0 \%$ ), radial length (accuracy $71.0 \%$ ), roughness (accuracy 74.2\%), smoothness (accuracy 77.2\%), and speculation (accuracy 72.6\%) for morphological features. Linear combination of all morphological and/or of all dynamic features did not increase accuracy in metastatic lymph nodes discrimination. Conclusions. Compactness as morphological feature and area under time-intensity curve as dynamic feature were the best parameters in identifying metastatic lymph nodes on breast MRI.
\end{abstract}

\section{Background}

In 2017, breast cancer had the highest incidence among female cancers and is still the second (after lung) leading cause of death from cancer in the US [1]. The transition from nonmetastatic to metastatic state of breast cancer is characterised by the diffusion of the primary lesion towards lymphatic sites. Therefore, accurate evaluation of metastasis in axillaries lymphatic nodes is a crucial factor affecting medical management, surgery, and prognosis [2-4]. 
Sentinel lymph-node biopsy (SLNB) has been effectively used for identifying, via radiotracer and/or blue dye, the nodes draining the breast which are possibly the first to be encountered during tumor spreading [5]. SLNB is commonly executed after surgical removal of the primary lesion and has shown an accuracy of 93.5 to $97.5 \%[6,7]$. However, it has been noticed that SLNB can have long-term morbidity that potentially can affect the quality of life despite being less significant than axillary lymph-node dissection $[8,9]$. In addition, preoperative evaluation of axillary lymph nodes might improve patient-based treatment: in fact, options might include neoadjuvant chemotherapy, intraoperative breast radiotherapy, and reconstruction planning. Moreover, when metastatic axillary disease is diagnosed before surgery, the surgeon can discuss specific aspects of axillary lymphnode dissection with the patient.

Despite being not very accurate, imaging techniques such as ultrasound (US), computed tomography (CT), and positron emission tomography (PET)/CT are often used in clinical practice [10-13]. Breast magnetic resonance imaging (MRI), because of its versatility, has gained a large consensus over the past two decades and many technological improvements have contributed to its diffusion [12]. In particular, dynamic contrast-enhanced magnetic resonance imaging (DCE-MRI) has been shown to be able to distinguish benign from malignant breast lesions by means of simultaneous evaluation of morphological and functional information. At the time of writing, axillary lymph nodes evaluation via DCEMRI has not yet been introduced in clinical practice. Mainly, diagnostic criteria for malignancy of axillary lymph nodes are based exclusively on morphology; however, these are still controversial $[11,14,15]$.

In this study, we investigated the discriminative power of MRI in both morphological and functional features derived by dynamic contrast-enhanced MRI (DCE-MRI) for axillary lymph-node evaluation. We attempted to identify the best quantitative feature to discriminate metastatic from nonmetastatic lymph nodes among 26 morphological and functional parameters and their linear combinations.

\section{Methods}

2.1. Patients Inclusion Criteria. A prospectively collected database has been reviewed after Institutional Review Board approval. We identified 268 consecutive patients from February 2009 to December 2013 for newly diagnosed breast carcinoma. All these subjects had undergone DCE-MRI in a single cancer centre. The study population comprised 52 patients with breast cancer who also underwent pathological evaluation of axillary lymph nodes. Age ranged from 31 to 58 years. Patients included in the study (1) had breast cancer with clinical evaluation (TNM score) T1-T2 and (2) underwent SLNB or/and axillary lymphadenectomy. Patients who carried an implanted device, were pregnant, or had any contraindication for MRI were not included in the study. In addition, we excluded patients having undergone radiation therapy or chemotherapy within 12 months before the MRI. All patients provided informed consent to the use of their data for research purposes. This retrospective study was performed according to regulations issued by our local Institutional Review Board.

2.2. MRI Methodology. DCE-MRI has been executed using $1.5 \mathrm{~T}$ breast-dedicated equipment (Aurora; Aurora Imaging Technology, North Andover, USA), embodying an in-table coil [14]. Exams were arranged from the 7th to 14th day of the menstrual cycle in premenopausal women; no scheduling limitations were applied in postmenopausal women.

The sequence used for precontrast imaging was a threedimensional (3D) nonspoiled SPIRAL-RODEO fat-sat (TR $29 \mathrm{~ms}$, TE $4.8 \mathrm{~ms}$, flip angle $45^{\circ}$, matrix $512 \times 512$, thickness $1.13 \mathrm{~mm}$, and gap $1.13 \mathrm{~mm}$ ); after contrast injection, four dynamic 3D spoiled SPIRAL-RODEO fat-sat acquisitions (TR $29 \mathrm{~ms}$, TE $4.8 \mathrm{~ms}$, flip angle $45^{\circ}$, matrix $512 \times 512$, thickness $1.13 \mathrm{~mm}$, and gap $1.13 \mathrm{~mm}$ ) were used. The time interval between acquisitions was $90 \mathrm{~s}$. A bolus of gadobenate dimeglumine (Multihance, Gd-BOPTA Bracco; Atlanta Pharma, Konstanz, Germany) has been intravenously injected using a dose of $0.1 \mathrm{mmol} / \mathrm{kg}$ body weight at a flow rate of $2 \mathrm{ml} / \mathrm{s}$, followed by $20 \mathrm{ml}$ of saline solution at the same rate. An automatic contrast delivery system was employed (Optistar Elite, Covidien Imaging Solution, Hazelwood, USA).

2.3. Histopathological Evaluation and Operation of Axillary Lymph Nodes. Samples of SLNB were assessed by immediate frozen section and hematoxylin and eosin staining. The nodes were subsequently submitted for permanent sectioning and immunohistochemical assay. According to the American Joint Committee on Cancer guidelines for breast cancer staging [16], a patient with isolated tumor cells was considered node-negative and did not undergo any additional lymphnode surgery.

2.4. Images Analysis. Two radiologists having more than 15 years of experience (AP) and more than 10 years of experience (SF), respectively, reviewed images. For each lymph node having a lower diameter $\geq 10 \mathrm{~mm}$, the manual segmentation was made using OsiriX v.3.8.1, on the data acquired after contrast injection using a pulse sequence for three-dimensional fat-saturated axial nonspoiled SPIRALRODEO images (Figure 1). Per each lymph node, on each slice, a region of interest (ROI) was drawn: the set of all ROIs corresponding to a single lymph node formed a Volume of Interest (VOI). ROI border has been placed in the lymphnode periphery close to the margin. Lymph nodes were evaluated using quantitative descriptors involving morphological and dynamic parameters.

2.5. Dynamic Parameters. Nine dynamic features emerged from the literature [17-20], which were extracted using the approach previously reported in a previous publication from our group [19] (Figure 2): maximum signal difference (MSD), the time to peak (TTP) between the wash-in (WI) and washout (WO) segments, the WI slope (WIS), the WO slope (WOS), the WI intercept (WII), the WO intercept (WOI), the area under curve (AUC), the area under WI tract (AUCWI), and the area under WO tract (AUCWO). 


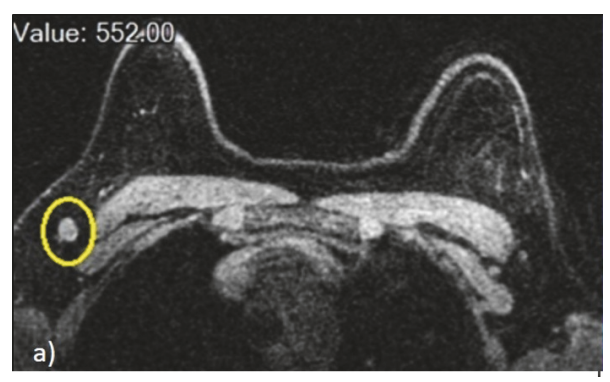

(a)

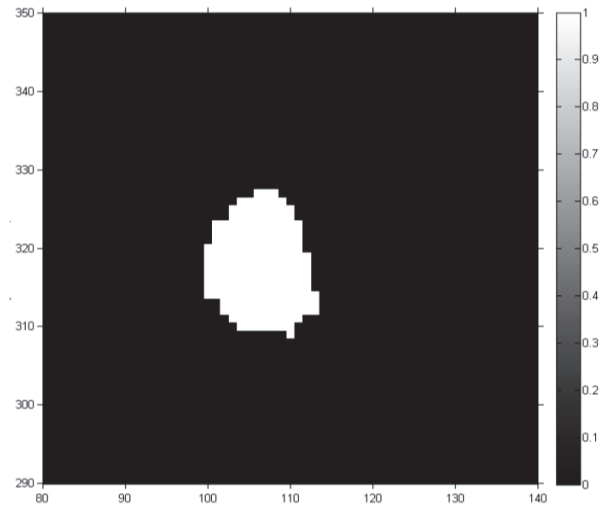

(c)

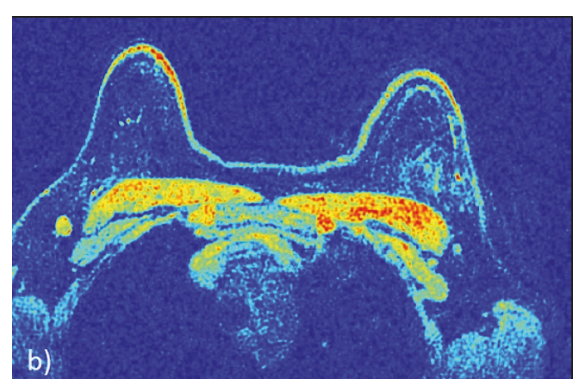

(b)

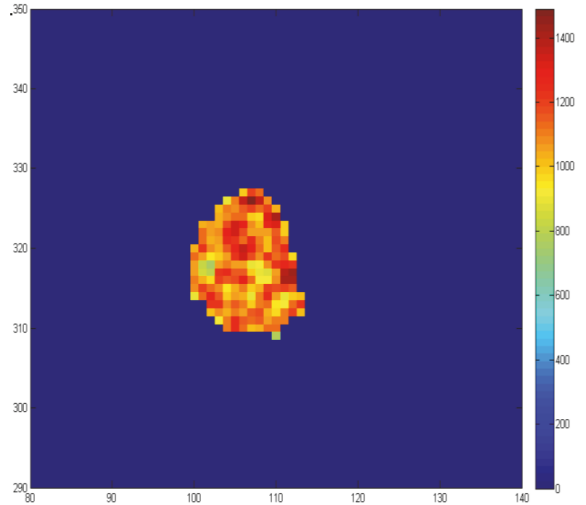

(d)

FIGURE 1: Lymph-node illustration on contrast-enhanced MR imaging and their segmentation for a single slice: (a)-(c) grey level; (b)-(d) RGB values.

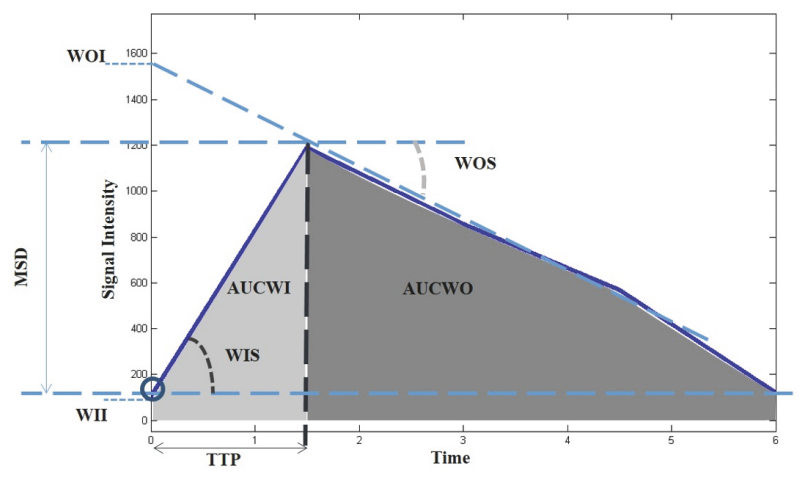

Figure 2: Dynamic parameters illustration.

2.6. Feature Extraction. Per each VOI, 17 morphological features were calculated [21-23]. Before feature computation, all lymph-node binary masks have been reinterpolated on a common grid of equal size $\left(1 \times 1 \times 1 \mathrm{~mm}^{3}\right)$ in three orthogonal directions. A brief description of all morphological features has been provided in Table 1. Detailed mathematical definitions might slightly vary among studies; therefore, we report the specific definitions we used:

(1) Circularity = (volume of the sphere with average lymph-node radius)/(lymph-node volume).

(2) Compactness $=($ lymph-node surface area $) /($ lymphnode volume).
(3) Irregularity $=1$ - (surface area of the sphere with average lymph-node radius)/(lymph-node surface area).

(4) Diameter $=$ diameter of the sphere corresponding to the lymph-node volume.

(5) Rectangularity $=$ (lymph-node volume $) /($ volume of the smallest parallelepiped containing the lymph node).

(6) Radial length = average distance between boundary points and lymph-node barycentre.

(7) Volume $=$ number of voxels of the lymph node times the volume of a single voxel.

(8) Smoothness $=(1 / N) \sum_{n} R_{n}-\left(R_{n-1}+R_{n+1}\right) / 2$, where $R n$ is the $n$th boundary point distance from the barycentre along a lymph-node slice.

(9) Curvature $=$ average $\left(\operatorname{abs}\left(x^{\prime} y^{\prime \prime}-y^{\prime} x^{\prime \prime}\right) /\left(x^{\prime 2}+y^{\prime 2}\right)^{(3 / 2)}\right)$, where $x, y$ are the coordinate parametric representation of the boundary points along a lymph-node slice, $x^{\prime} y^{\prime}$ are the first derivative with respect to the parameter, and $x^{\prime \prime} y^{\prime \prime}$ are the second derivative.

(10) Roughness: $\left(\left[(1 / N) \sum_{n=1}^{N}\left(R_{n}-\mu\right)^{4}\right]^{1 / 4}-[(1 /\right.$ N) $\left.\left.\sum_{n=1}^{N}\left(R_{n}-\mu\right)^{2}\right]^{1 / 2}\right) / \mu$, where $N$ is the number of points of the boundary, $R n$ is the radial distance of the $n$th point, and $\mu$ is the average radial distance.

(11) Sphericity $=$ (average radial length $) /($ standard deviation radial length). 
TABLE 1: List of features used in analysis with definitions.

\begin{tabular}{|c|c|c|}
\hline Feature category & Feature & Description \\
\hline \multirow{9}{*}{ Dynamic } & TTP & Time to peak \\
\hline & MSD & Maximum signal difference \\
\hline & AUGC & Area under gadolinium curve \\
\hline & AUCWI & Area under gadolinium curve in the wash-in phase \\
\hline & AUCWO & Area under gadolinium curve in the wash-out phase \\
\hline & WIS & Wash-in slope \\
\hline & WII & Wash-in intercept \\
\hline & WOS & Wash-out slope \\
\hline & WOI & Wash-out intercept \\
\hline \multirow{17}{*}{ Morphological } & circularity & Similarity of the lesion shape to a sphere \\
\hline & compactness & Ratio between surface and volume \\
\hline & convexity & $\begin{array}{l}\text { Ratio between the smallest volume with convex } \\
\text { curvature that contains the lymph node and its volume }\end{array}$ \\
\hline & curvature & Measure of curvature of lymph node contour \\
\hline & elongation & $\begin{array}{l}\text { Parameter that estimates how much the ROI is } \\
\text { pronounced along one direction than along the other }\end{array}$ \\
\hline & diameter & Diameter of the sphere having the same ROI volume \\
\hline & eccentricity & $\begin{array}{c}\text { Ratio of the larger rope and the largest among the } \\
\text { orthogonal ropes }\end{array}$ \\
\hline & irregularity & $\begin{array}{l}\text { Deviation of the lesion surface from the surface of a } \\
\text { sphere }\end{array}$ \\
\hline & radial length & $\begin{array}{l}\text { Average distance between points on the border and the } \\
\text { center of the lymph node }\end{array}$ \\
\hline & entropy & Entropy of radial length \\
\hline & rectangularity & Similarity of the lesion shape to a rectangle \\
\hline & roughness & $\begin{array}{c}\text { Distances of each point of the center than the radial } \\
\text { length average }\end{array}$ \\
\hline & smoothness & Measurement of lymph node contour irregularities \\
\hline & sphericity & $\begin{array}{l}\text { Ratio between the average radial length and the } \\
\text { standard deviation of the rays }\end{array}$ \\
\hline & spiculation & $\begin{array}{l}\text { Standard deviation of the radial lengths with respect to } \\
\text { the radial length average }\end{array}$ \\
\hline & surface & Sum of lymph nodes contour pixels \\
\hline & volume & Volume of the entire lymph node \\
\hline
\end{tabular}

(12) Eccentricity = (lymph-node largest diameter $) /($ the largest diameter orthogonal to the previous one).

(13) Surface $=$ number of voxels belonging to the lymphnode boundary.

(14) Spiculation = standard deviation of radial length.

(15) Convexity $=($ convex-hull volume $) /($ lymph-node volume);

(16) Entropy: $-\sum_{n} P\left(R_{n}=r_{n}\right) \log P\left(R_{n}=r_{n}\right)$, where $P(R=r n)$ is the distribution of radial length.

(17) Elongation $=($ length $) /($ width $)$ of the smallest rectangle containing the lymph node averaged per each slice in three orthogonal directions.

2.7. Statistical Analysis. Histopathological results after surgical intervention served as a reference standard for $N$ staging. For each parameter, median and standard deviation (SD) were calculated as representative values of segmented VOI. Interobserver agreement was calculated to assess the variability between two readers in the manual lymph-nodes segmentation. As is commonly reported, an interobserver correlation coefficient of $0-0.20$ reflected a poor agreement, of 0.21-0.40 reflected a fair agreement, of 0.41-0.60 reflected a moderate agreement, of $0.61-0.80$ reflected a good agreement, and of $0.81-1.00$ reflected an excellent agreement. The nonparametric Mann-Whitney test was used to emphasize statistically significant difference between median values of morphological and dynamic parameters in metastatic lymphnodes group versus nonmetastatic lymph-nodes group. A $P$ value of $<0.05$ was considered significant for all tests.

Receiver operating characteristic (ROC) analysis in addition to sensitivity, specificity, misclassification error (number 
TABLE 2: Morphological features median and standard deviation values for each parameter. $P$ value was calculated using Mann-Whitney test.

\begin{tabular}{|c|c|c|c|c|c|}
\hline \multirow{3}{*}{ Morphological Features } & \multirow{2}{*}{\multicolumn{2}{|c|}{$\begin{array}{c}\text { Metastatic } \\
\text { Lymph-nodes }\end{array}$}} & \multicolumn{2}{|c|}{ Non Metastatic } & \multirow{3}{*}{$P$ value } \\
\hline & & & \multicolumn{2}{|c|}{ Lymph-nodes } & \\
\hline & Median & SD & Median & SD & \\
\hline circularity & 0,001 & 0,002 & 0,002 & 0,003 & 0,829 \\
\hline compactness & 0,339 & 0,125 & 0,453 & 0,076 & 0,021 \\
\hline Convexity & 0,824 & 0,133 & 0,857 & 0,159 & 0,005 \\
\hline curvature & 0,091 & 0,038 & 0,133 & 0,052 & 0,001 \\
\hline Elongation & 1,133 & 0,307 & 1,281 & 0,430 & 0,000 \\
\hline diameter & 17,812 & 7,891 & 14,392 & 3,278 & 0,000 \\
\hline eccentricity & 1,658 & 0,584 & 1,679 & 0,678 & 0,000 \\
\hline Irregularity & $-3,487$ & 0,562 & $-3,425$ & 0,760 & 0,000 \\
\hline radial length & 0,929 & 0,031 & 0,960 & 0,020 & 0,000 \\
\hline Entropy & 4,763 & 0,214 & 4,664 & 0,241 & 0,000 \\
\hline rectangularity & 0,358 & 0,071 & 0,332 & 0,092 & 0,000 \\
\hline Roughness & 0,353 & 0,232 & 0,102 & 0,078 & 0,000 \\
\hline smoothness & 4,283 & 1,914 & 3,320 & 0,539 & 0,000 \\
\hline sphericity/roundness & 26,372 & 15,870 & 41,890 & 27,259 & 0,000 \\
\hline Spiculation & 6,602 & 2,656 & 4,323 & 1,369 & 0,000 \\
\hline Surface & 1094,000 & 1207,675 & 698,000 & 540,839 & 0,000 \\
\hline volume & 2959,000 & 7956,682 & 1561,000 & 1114,509 & 0,000 \\
\hline
\end{tabular}

TABle 3: Dynamic features median and standard deviation values for each parameter. $P$ value was calculated using Mann-Whitney test.

\begin{tabular}{|c|c|c|c|c|c|}
\hline \multirow{2}{*}{ Features } & \multicolumn{2}{|c|}{ Metastatic Lymph-nodes } & \multicolumn{2}{|c|}{ Non Metastatic Lymph-nodes } & \multirow{2}{*}{$P$ value } \\
\hline & Median & SD & Median & $\mathrm{SD}$ & \\
\hline MSD & 1079,000 & 641,773 & 597,500 & 474,324 & 1,000 \\
\hline TTP & 372,261 & 169,279 & 459,888 & 179,981 & 0,021 \\
\hline AUC & 3,000 & 1,193 & 3,000 & 0,994 & 0,003 \\
\hline WII & 1,453 & 0,215 & 1,521 & 0,198 & 0,001 \\
\hline WOI & 11475,000 & 3002,216 & 8871,375 & 2198,225 & 0,000 \\
\hline WIS & 1924,637 & 819,422 & 2005,110 & 668,567 & 0,037 \\
\hline WOS & 1384,000 & 535,408 & 1442,450 & 486,190 & 0,214 \\
\hline AUCWI & 395,952 & 174,820 & 468,695 & 179,586 & 0,002 \\
\hline AUCWO & 2951,250 & 1549,637 & 3654,000 & 1280,761 & 0,000 \\
\hline
\end{tabular}

of false negatives and false positives over the total), and accuracy (number of true negatives and true positives over the total) has been performed with respect to histopathological results.

Moreover, we applied a linear discriminant analysis (LDA) [24] to identify the best weighted linear combination of features producing the best results considering, respectively, morphological features only, dynamic features only, and both kinds of features together (sensibility and specificity were reported and were considered significant for the features with an accuracy of $>70 \%$ at ROC analysis). 10 -fold crossvalidation has been performed in order to have robust result [24].

Statistical processing and classification have been performed by means of the Statistics Toolbox within Matlab R2007a (MathWorks Inc., Natick, USA).

\section{Results}

In the present study, 94 dominant lymph nodes were evaluated in 52 patients with primary breast carcinoma: 48 metastatic lymph nodes and 46 not pathological lymph nodes.

Table 2 reports median and standard deviation for each morphological parameter in the metastatic lymph-nodes group versus the nonmetastatic lymph-nodes group. The median of all the parameters, except circularity, showed a statistically significant difference between the two groups.

Table 3 reports median and standard deviation for each dynamic parameter in the metastatic lymph-nodes group versus the nonmetastatic lymph-nodes group. The median of all parameters, except MSD and WOS, showed a statistically significant difference between the two groups. 
TABLE 4: ROC analysis findings for each morphological and dynamic parameter in terms of sensitivity, specificity, misclassification error, and accuracy.

\begin{tabular}{|c|c|c|c|c|c|}
\hline Features & Sensitivity [\%] & Specificity [\%] & Misclassification Error [\%] & Accuracy [\%] & AUC \\
\hline MSD & 73,910 & 64,100 & 32,260 & 67,740 & 0.668 \\
\hline TTP & 38,890 & 34,620 & 62,900 & 37,100 & 0.516 \\
\hline AUC & 80,770 & 72,220 & 24,190 & 75,810 & 0.769 \\
\hline WII & 40,000 & 43,240 & 58,060 & 41,940 & 0.501 \\
\hline WOI & 44,830 & 45,450 & 54,840 & 45,160 & 0.437 \\
\hline WIS & 78,260 & 66,670 & 29,030 & 70,970 & 0.717 \\
\hline WOS & 70,970 & 70,970 & 29,030 & 70,970 & 0.657 \\
\hline AUCWI & 28,570 & 39,020 & 64,520 & 35,480 & 0.459 \\
\hline AUCWO & 81,820 & 67,500 & 27,420 & 72,580 & 0.766 \\
\hline Circularity & 60,980 & 71,430 & 35,480 & 64,520 & 0.762 \\
\hline Compactness & 83,330 & 81,250 & 17,740 & 82,260 & 0.824 \\
\hline Convexity & 42,420 & 41,380 & 58,060 & 41,940 & 0.469 \\
\hline Curvature & 68,570 & 74,070 & 29,030 & 70,970 & 0.770 \\
\hline Elongation & 57,890 & 62,500 & 40,320 & 59,680 & 0.680 \\
\hline Diameter & 69,230 & 63,890 & 33,870 & 66,130 & 0.738 \\
\hline Eccentricity & 52,630 & 54,170 & 46,770 & 53,230 & 0.453 \\
\hline Irregularity & 54,550 & 55,170 & 45,160 & 54,840 & 0.448 \\
\hline Radial Length & 76,000 & 67,570 & 29,030 & 70,970 & 0.811 \\
\hline Entropy & 62,500 & 72,730 & 33,870 & 66,130 & 0.699 \\
\hline Rectangularity & 56,670 & 56,250 & 43,550 & 56,450 & 0.564 \\
\hline Roughness & 85,710 & 68,290 & 25,810 & 74,190 & 0.834 \\
\hline Smoothness & 90,480 & 70,730 & 22,580 & 77,420 & 0.810 \\
\hline Sphericity/roundness & 62,790 & 78,950 & 32,260 & 67,740 & 0.710 \\
\hline Spiculation & 81,820 & 67,500 & 27,420 & 72,580 & 0.742 \\
\hline Surface & 68,180 & 60,000 & 37,100 & 62,900 & 0.713 \\
\hline Volume & 92,860 & 62,500 & 30,650 & 69,350 & 0.738 \\
\hline
\end{tabular}

TABLE 5: LDA analysis findings when all morphological and dynamic features were considered and when the linear combinations of significant morphological and dynamic features were considered.

\begin{tabular}{lccc}
\hline & Sensitivity [\%] & Specificity [\%] & AUC \\
\hline All dynamic features & 77,420 & 70,970 & 0.778 \\
All morphological features & 70,970 & 80,650 & 0.803 \\
All features & 64,520 & 77,400 & 0.754 \\
All significant dynamic features & 85,000 & 66,700 & 0.794 \\
All significant morphologic features & 88,500 & 77,800 & 0.812 \\
All significant features & 81,000 & 65,900 & 0.789 \\
\hline
\end{tabular}

Interobserver correlation coefficient calculated on VOI for each segmented lymph node was of 0.864 (95\% CI: 0.835-0.884) indicating an excellent agreement between the two manual segmentations; in addition, this indicates the robustness of morphological and dynamic parameters calculated on segmented lymph nodes.

Table 4 reports findings of ROC analysis for each morphological and dynamic parameter in terms of sensitivity, specificity, misclassification error, and accuracy. The best discrimination between metastatic lymph nodes and nonmetastatic lymph nodes has been obtained by AUC, WIS, WOS, and AUCWO of the dynamic features and by compactness, curvature, radial length, roughness, smoothness, and speculation of the morphological features.

Table 5 reports the finding of LDA analysis when all morphological and dynamic features were considered and when the linear combination of significant morphological and dynamic features was considered.

\section{Discussion}

Histopathologic staging of axillary lymph node is one of the most commonly used predictors of breast cancer survival. Currently, diagnosis of metastatic involvement requires 
invasive procedures such as pathologic assessment of biopsy tissue or postsurgery dissection. Conventional MRI with double breast coils can noninvasively evaluate both breasts and simultaneously assess axillary lymph nodes; moreover, new techniques, such as DCE-MRI, are now achieving a sufficient degree of maturity for breast cancer evaluation. The verification of MRI-based diagnoses of a specific node with histopathologic analysis of the same node is still a challenge. Moreover, the use of DCE-MRI for assessment of metastatic axillary lymph nodes has not yet been sufficiently investigated and conflicting results until now have been published [2532]. In this study, we used several morphological features and several dynamic MRI characteristics of axillary lymph nodes. We investigated whether and how malignant nodes could be assessed preoperatively and noninvasively by means of MRI using both morphologic and dynamic criteria. The sensitivity of these features ranged from $28.6 \%$ to $92.9 \%$, and the specificity ranged from $34.6 \%$ to $81.3 \%$. The best results for discrimination of nonmetastatic lymph nodes by metastatic lymph nodes have been achieved by AUC, WIS, WOS, and AUCWO, among the dynamic features, and by compactness, curvature, radial length, roughness, smoothness, and speculation, among morphological features. The best dynamic parameter was AUC reporting a sensitivity, specificity, misclassification error, and accuracy of $81 \%, 72 \%$, $24 \%$, and $76 \%$, respectively. The best morphological parameter was compactness reporting a sensitivity, specificity, misclassification error, and accuracy of $83 \%, 81 \%, 18 \%$, and $82 \%$, respectively.

Our results are similar to those of other researchers [2529]. Choi et al. [25] performed a meta-analysis reporting the diagnostic performance of CT, MRI, and PET/CT for detection of metastatic lymph nodes in cervical cancer patients: for region- and node-based data analysis, MRI sensitivity and specificity were $38 \%$ and $97 \%$, respectively. He et al. [26] and Baltzer et al. [27] investigated diagnostic performance of specific morphological and/or dynamic features obtained by MR imaging. He et al. reported the area under ROC (AUROC) for short and long lymph-node axis of 0.89 and 0.74 , respectively (sensitivity of $93.3 \%$ and specificity of $72.6 \%$ for short axis, sensitivity of $88.1 \%$ and specificity of $64.1 \%$ for long axis) and the AUROC of early stage enhancement rate as a dynamic feature (sensitivity of $97.0 \%$ and specificity of $73.5 \%$ ). Baltzer et al. [27] investigated only the margin of lymph nodes as a morphological parameter reporting a sensitivity of $41.2 \%$ and a specificity of $95.2 \%$. Schacht et al. [28] reported the results of a quantitative breast MR image analysis for classification of axillary lymph nodes. The best features in that study were the circularity as a morphological parameter with AUROC of 0.67 and the wash-out rate with AUROC of 0.62 . Harada et al. [29] evaluated the diagnostic performance of morphologic features computable from MR images using a contrast agent actually not commercialized (ultrasmall superparamagnetic iron oxide): sensitivity, specificity, and overall accuracy were $36.5 \%, 94.1 \%$, and $81 \%$, respectively.

On the basis of our results, a linear combination of morphological and dynamic feature does not increase the accuracy in lymph-nodes discrimination. LDA results of each group and combination of groups (morphological and/or dynamic parameters) were comparable to results of dynamic and morphological parameters considered separately. A future endpoint could be to perform multivariate analysis of functional parameters including other modalities such as PET/CT examination or quantitative parameters derived by hybrid system like PET/MRI [33].

A limit of our study consists of the manual segmentation of lymph nodes. However, an expert breast radiologist performed this procedure. A second limitation is the level of complexity in the "gold standard" due to the difficult task of identifying which lymph nodes were biopsied or dissected and of matching the pathologic results to the imaged nodes.

\section{Disclosure}

Each author has participated sufficiently in the submission to take public responsibility for its content.

\section{Conflicts of Interest}

The authors have no conflicts of interest to disclose.

\section{References}

[1] https://www.cancer.org/content/dam/cancer-org/research/ cancer-facts-and-statistics/annual-cancer-facts-and-figures/ 2017/cancer-facts-and-figures-2017.pdf.

[2] G. Le Bouedec, T. Gauthier, P. Gimbergues, and J. Dauplat, "Axillary recurrence after negative sentinel lymph node biopsy in breast cancer," La Presse Médicale, vol. 37, no. 11, pp. 16851687, 2008.

[3] P. Strnad, L. Rob, H. Krízová, A. Zuntová, J. Chod, and M. Halaska, "Sentinel lymphatic node biopsy for breast cancer in practice," Česká Gynekologie, vol. 70, no. 4, pp. 291-295, 2005.

[4] S. W. Beenken, M. M. Urist, Y. Zhang et al., "Axillary Lymph Node Status, but Not Tumor Size, Predicts Locoregional Recurrence and Overall Survival after Mastectomy for Breast Cancer," Annals of Surgery, vol. 237, no. 5, pp. 732-739, 2003.

[5] S. A. McLaughlin, M. J. Wright, K. T. Morris et al., "Prevalence of lymphedema in women with breast cancer 5 years after sentinel lymph node biopsy or axillary dissection: Objective measurements," Journal of Clinical Oncology, vol. 26, no. 32, pp. 5213-5219, 2008.

[6] U. Veronesi, G. Paganelli, G. Viale et al., "A randomized comparison of sentinel-node biopsy with routine axillary dissection in breast cancer," The New England Journal of Medicine, vol. 349, no. 6, pp. 546-553, 2003.

[7] D. N. Krag, S. J. Anderson, T. B. Julian et al., “Technical outcomes of sentinel-lymph-node resection and conventional axillary-lymph-node dissection in patients with clinically nodenegative breast cancer: results from the NSABP B-32 randomised phase III trial," The Lancet Oncology, vol. 8, no. 10, pp. 881-888, 2007.

[8] R. Crane-Okada, R. A. Wascher, D. Elashoff, and A. E. Giuliano, "Long-term morbidity of sentinel node biopsy versus complete axillary dissection for unilateral breast cancer," Annals of Surgical Oncology, vol. 15, no. 7, pp. 1996-2005, 2008.

[9] A. D. Purushotham, S. Upponi, M. B. Klevesath et al., "Morbidity after sentinel lymph node biopsy in primary breast cancer: results from a randomized controlled trial," Journal of Clinical Oncology, vol. 23, no. 19, pp. 4312-4321, 2005. 
[10] P. Vassallo, G. Edel, N. Roos, A. Naguib, and P. E. Peters, "Invitro high-resolution ultrasonography of benign and malignant lymph nodes: A sonographic-pathologic correlation," Investigative Radiology, vol. 28, no. 8, pp. 698-705, 1993.

[11] G. C. Dooms, H. Hricak, L. E. Crooks, and C. B. Higgins, "Magnetic resonance imaging of the lymph nodes: Comparison with CT," Radiology, vol. 153, no. 3 I, pp. 719-728, 1984.

[12] S. Ueda, H. Tsuda, H. Asakawa et al., "Utility of $18 \mathrm{~F}-$ fluorodeoxyglucose emission tomography/ computed tomography fusion imaging (18F-FDG PET/CT) in combination with ultrasonography for axillary staging in primary breast cancer," $B M C$ Cancer, vol. 8, article no. 165, 2008.

[13] K. L. Cooper, Y. Meng, S. Harnan et al., "Positron emission tomography (PET) and magnetic resonance imaging (MRI) for the assessment of axillary lymph node metastases in early breast cancer: systematic review and economic evaluation.", Health Technology Assessment, vol. 15, no. 4, 2011.

[14] M. Memarsadeghi, C. C. Riedl, A. Kaneider et al., "Axillary lymph node metastases in patients with breast carcinomas: assessment with nonenhanced versus USPIO-enhanced MR imaging," Radiology, vol. 241, no. 2, pp. 367-377, 2006.

[15] A. Luciani, F. Pigneur, F. Ghozali et al., "Ex vivo MRI of axillary lymph nodes in breast cancer," European Journal of Radiology, vol. 69, no. 1, pp. 59-66, 2009.

[16] S. B. Edge, D. R. Byrd, C. C. Compton, A. G. Fritz, F. L. Greene, and A. Trotti, American Joint Commision on Cancer. Breast Cancer: Prespectives on Anatomic Staging-Based on The AJCC Staging Manual, Springer, New York, NY, USA, 7th edition, 2010.

[17] R. Fusco, M. Sansone, S. Filice et al., "Integration of DCEMRI and DW-MRI Quantitative Parameters for Breast Lesion Classification," BioMed Research International, vol. 2015, Article ID 237863, 12 pages, 2015.

[18] T. Schlossbauer, G. Leinsinger, A. Wismuller et al., "Classification of small contrast enhancing breast lesions in dynamic magnetic resonance imaging using a combination of morphological criteria and dynamic analysis based on unsupervised vectorquantization," Investigative Radiology, vol. 43, no. 1, pp. 56-64, 2008.

[19] R. Fusco, A. Petrillo, M. Petrillo, and M. Sansone, "Use of tracer kinetic models for selection of semi-quantitative features for DCE-MRI data classification," Applied Magnetic Resonance, vol. 44, no. 11, pp. 1311-1324, 2013.

[20] W. J. Krzanowski, Principles of multivariate analysis, vol. 3 of Oxford Statistical Science Series, The Clarendon Press, Oxford University Press, New York, 1988.

[21] D. M. Ikeda, N. M. Hylton, K. Kinkel et al., "Development, standardization, and testing of a lexicon for reporting contrastenhanced breast magnetic resonance imaging studies," Journal of Magnetic Resonance Imaging, vol. 13, no. 6, pp. 889-895, 2001.

[22] C. E. McLaren, W.-P. Chen, K. Nie, and M.-Y. Su, "Prediction of Malignant Breast Lesions from MRI Features. A Comparison of Artificial Neural Network and Logistic Regression Techniques," Academic Radiology, vol. 16, no. 7, pp. 842-851, 2009.

[23] M. Sansone, R. Fusco, A. Petrillo, M. Petrillo, and M. Bracale, "An expectation-maximisation approach for simultaneous pixel classification and tracer kinetic modelling in dynamic contrast enhanced-magnetic resonance imaging," Medical \& Biological Engineering \& Computing, vol. 49, no. 4, pp. 485-495, 2011.
[24] R. Fusco, M. Sansone, S. Filice et al., "Pattern Recognition Approaches for Breast Cancer DCE-MRI Classification: A Systematic Review," Journal of Medical and Biological Engineering, vol. 36, no. 4, pp. 449-459, 2016.

[25] H. J. Choi, W. Ju, S. K. Myung, and Y. Kim, "Diagnostic performance of computer tomography, magnetic resonance imaging, and positron emission tomography or positron emission tomography/computer tomography for detection of metastatic lymph nodes in patients with cervical cancer: Meta-analysis," Cancer Science, vol. 101, no. 6, pp. 1471-1479, 2010.

[26] N. He, C. Xie, W. Wei et al., "A new, preoperative, MRI-based scoring system for diagnosing malignant axillary lymph nodes in women evaluated for breast cancer," European Journal of Radiology, vol. 81, no. 10, pp. 2602-2612, 2012.

[27] P. A. T. Baltzer, M. Dietzel, H. P. Burmeister et al., "Application of MR mammography beyond local staging: Is there a potential to accurately assess axillary lymph nodes? Evaluation of an extended protocol in an initial prospective study," American Journal of Roentgenology, vol. 196, no. 5, pp. W641-W647, 2011.

[28] D. V. Schacht, K. Drukker, I. Pak, H. Abe, and M. L. Giger, "Using quantitative image analysis to classify axillary lymph nodes on breast MRI: A new application for the Z 0011 Era," European Journal of Radiology, vol. 84, no. 3, pp. 392-397, 2015.

[29] T. Harada, N. Tanigawa, M. Matsuki, T. Nohara, and I. Narabayashi, "Evaluation of lymph node metastases of breast cancer using ultrasmall superparamagnetic iron oxideenhanced magnetic resonance imaging," European Journal of Radiology, vol. 63, no. 3, pp. 401-407, 2007.

[30] A. Luciani, T. H. Dao, M. Lapeyre et al., "Simultaneous Bilateral Breast and High-Resolution Axillary MRI of Patients with Breast Cancer: Preliminary Results," American Journal of Roentgenology, vol. 182, no. 4, pp. 1059-1067, 2004.

[31] S. O. Hwang, S.-W. Lee, H. J. Kim, W. W. Kim, H. Y. Park, and J. H. Jung, "The comparative study of ultrasonography, contrastenhanced MRI, and 18F-FDG PET/CT for detecting axillary lymph node metastasis in T1 breast cancer," Journal of Breast Cancer, vol. 16, no. 3, pp. 315-321, 2013.

[32] S. A. Valente, G. M. Levine, M. J. Silverstein et al., "Accuracy of predicting axillary lymph node positivity by physical examination, mammography, ultrasonography, and magnetic resonance imaging," Annals of Surgical Oncology, vol. 19, no. 6, pp. 18251830, 2012.

[33] S. G. Kim, K. Friedman, S. Patel, and M. Hagiwara, "Potential role of PET/MRI for imaging metastatic lymph nodes in head and neck cancer," American Journal of Roentgenology, vol. 207, no. 2, pp. 248-256, 2016. 


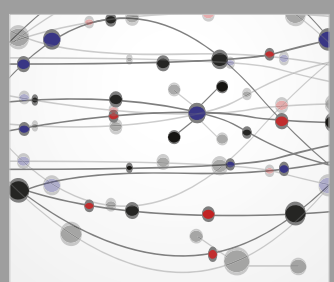

The Scientific World Journal
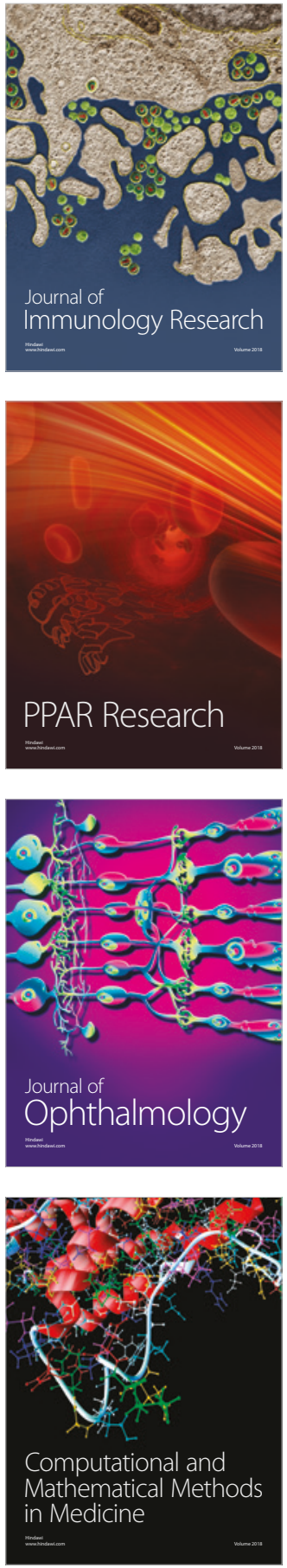

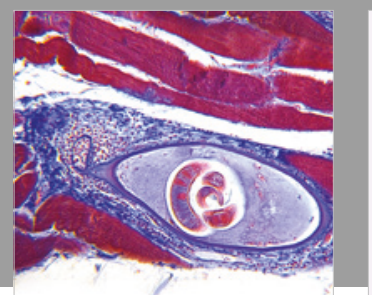

Gastroenterology Research and Practice

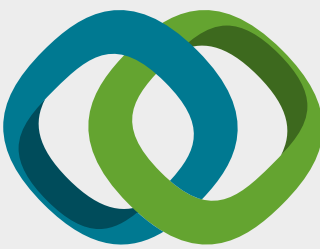

\section{Hindawi}

Submit your manuscripts at

www.hindawi.com
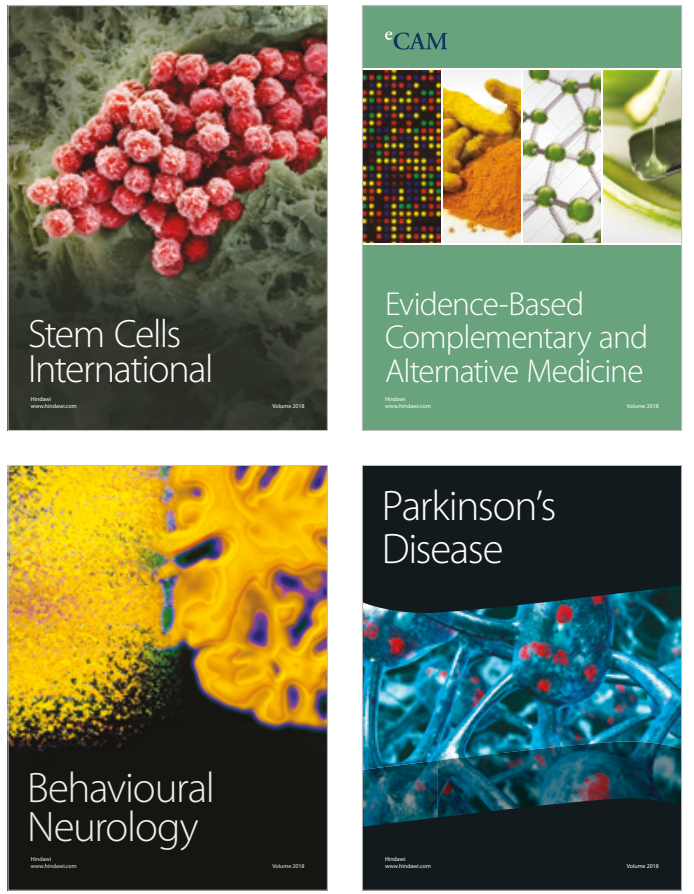

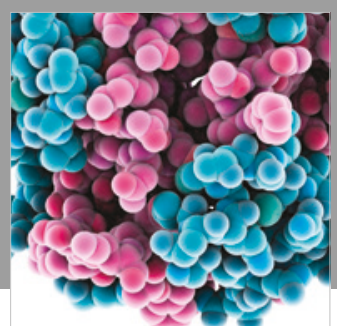

ournal of

Diabetes Research

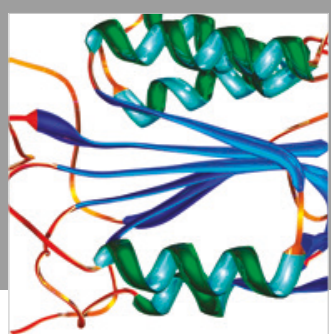

Disease Markers
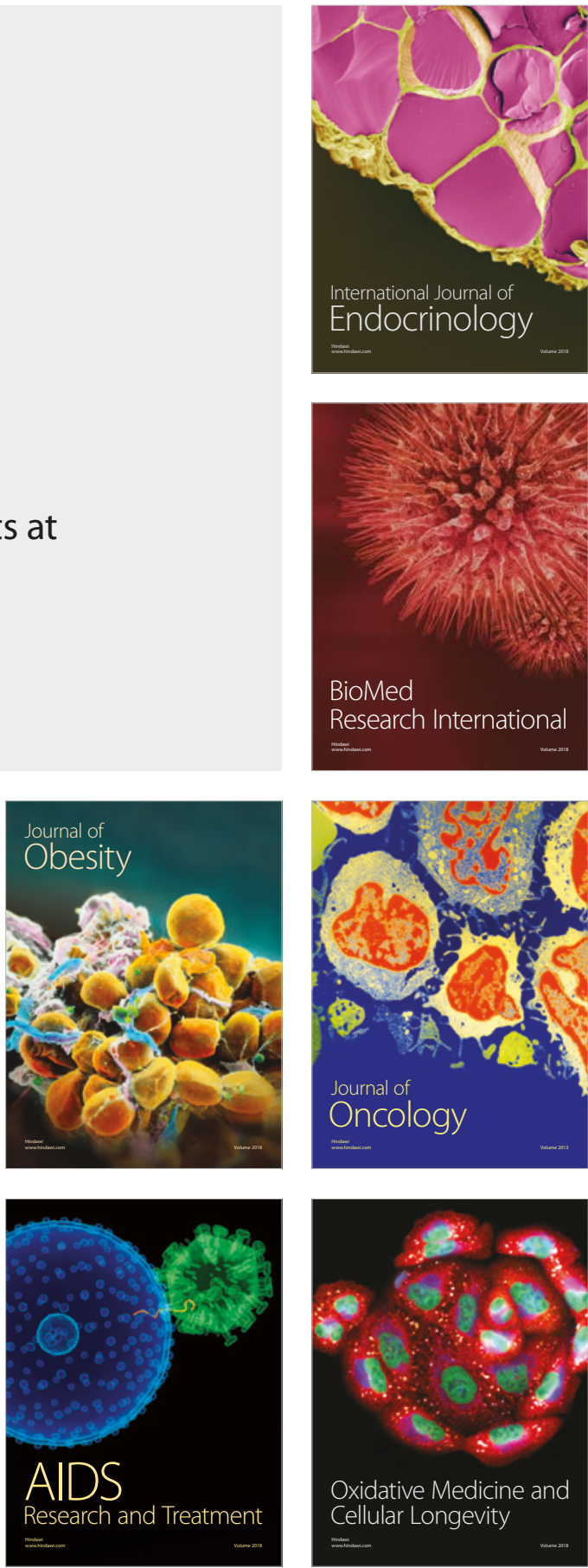